\title{
Systemic inflammation in glucocerebrosidase-deficient mice with minimal glucosylceramide storage
}

\author{
Hiroki Mizukami, ${ }^{1}$ Yide Mi, ${ }^{1}$ Ryuichi Wada, ${ }^{1,2}$ Mari Kono, ${ }^{1}$ Tadashi Yamashita, ${ }^{1}$ \\ Yujing Liu, ${ }^{1}$ Norbert Werth, ${ }^{3}$ Roger Sandhoff, ${ }^{4}$ Konrad Sandhoff, ${ }^{3}$ and Richard L. Proia ${ }^{1}$ \\ ${ }^{1}$ Genetics of Development and Disease Branch, National Institute of Diabetes and Digestive and Kidney Diseases, \\ National Institutes of Health, Bethesda, Maryland, USA \\ ${ }^{2}$ Department of Pathology, Hirosaki University School of Medicine, Hirosaki, Japan \\ ${ }^{3}$ Kekulé-Institut für Organische Chemie und Biochemie, Universität Bonn, Bonn, Germany \\ ${ }^{4}$ Deutsches Krebsforschungszentrum Heidelberg, Abteilung für Zelluläre und Molekulare Pathologie, Heidelberg, Germany \\ Address correspondence to: Richard L. Proia, Building 10, Room 9N-314, National Institutes of Health, \\ 10 Center DR MSC 1821, Bethesda, Maryland 20892-1821, USA. \\ Phone: (301) 496-4391; Fax: (301) 496-9878; E-mail: proia@nih.gov.
}

Received for publication October 30, 2001, and accepted in revised form March 29, 2002.

Gaucher disease, the most common lysosomal storage disease, is caused by a deficiency of glucocerebrosidase resulting in the impairment of glucosylceramide degradation. The hallmark of the disease is the presence of the Gaucher cell, a macrophage containing much of the stored glucosylceramide found in tissues, which is believed to cause many of the clinical manifestations of the disease. We have developed adult mice carrying the Gaucher disease L444P point mutation in the glucocerebrosidase (Gba) gene and exhibiting a partial enzyme deficiency. The mutant mice demonstrate multisystem inflammation, including evidence of $B$ cell hyperproliferation, an aspect of the disease found in some patients. However, the mutant mice do not accumulate large amounts of glucosylceramide or exhibit classic Gaucher cells in tissues.

J. Clin. Invest. 109:1215-1221 (2002). DOI:10.1172/JCI200214530.

\section{Introduction}

Gaucher disease, the most frequently occurring lysosomal storage disorder, is caused by mutations in the gene (GBA) encoding glucocerebrosidase, known also as acid $\beta$-glucosidase (reviewed in ref. 1). Deficiency of the enzyme impairs the lysosomal degradation of glycosphingolipids, leading to the accumulation of glucosylceramide primarily in macrophages. These storage macrophages, known as Gaucher cells, are believed to underlie many of the clinical manifestations of the disorder.

The most common form of Gaucher disease is type 1, which is distinguished from the other forms of the disease, types 2 and 3 , by the lack of neurologic involvement. Within type 1 Gaucher disease there is a broad spectrum of clinical severity ranging from patients with life-threatening manifestations in childhood to some individuals that are relatively asymptomatic throughout their lives. Enzyme replacement therapy is an effective treatment for many of the manifestations of type 1 disease (2-4). Another therapeutic approach, substrate deprivation, is focused on inhibiting synthesis of glucosylceramide with small-molecule inhibitors of glucosylceramide synthase, the enzyme responsible for the synthesis of glucosylceramide (5-8).

The clinical features in type 1 Gaucher disease are heterogeneous and affect many organ systems. The major disease manifestations can include massive hepatosplenomegaly, bone lesions, hematologic abnormalities, and lung involvement. The disease has also been reported to be associated with a sustained inflam- matory reaction (9). In this context some patients have been described with lymphadenopathy (1), polyclonal or monoclonal gammopathies (9-11), and elevated levels of proinflammatory cytokines (12-15). An increased risk for lymphoproliferative disorders involving the $\mathrm{B}$ cell lineage has also been reported $(9,10,16-19)$.

The complex pathophysiology of the disease is not well understood, in large part due to the absence of viable animal models. A complete disruption of the Gba gene in mice resulted in rapid neonatal death (20). In an attempt to produce a viable model we introduced a human Gaucher disease point mutation, L444P, into the mouse Gba gene to cause a partial enzyme deficiency. Although less severely affected than the knockout animals, the L444P mutant mice only survived for a few days after birth due to compromised epidermal barrier function (21). We have now developed a breeding scheme that allows long-term survival of some mice homozygous for the L444P mutation. Although these mice do not develop bulk accumulation of glucosylceramide in tissues or demonstrate Gaucher cells, they do display systemic inflammation including evidence of B cell hyperproliferation. The results indicate that $\beta$-glucocerebrosidase deficiency, even in the absence of large amounts of sphingolipid storage, can trigger an inflammatory reaction.

\section{Methods}

Generation of mutant mice. Previously we described mice with the human L444P point mutation in the Gba gene 
that were produced by a knock-in procedure using the mouse gene containing the mutation (21). We found that mice homozygous for the mutation died within 3 days after birth, presumably due to impaired permeability barrier function in the epidermis (21). This skin defect causes excessive transepidermal water loss, contributing to the demise of the pups (22). We have now been able to extend the lifespan of some mice homozygous for the mutation by a combination of optimized breeding conditions and improved animal husbandry.

We find that the type of cage bedding used is important for pup survival. Wood chip bedding supports some pup survival, whereas more highly absorbent beddings such as those produced from recycled paper or corn cobs generally do not. The highly absorbent beddings may exacerbate moisture loss from the mutant pups.

The second important factor in producing sufficient numbers of viable mutant mice was the establishment of homozygous $G b a^{L 444 P}$ breeding pairs. We were initially able to produce homozygous mutant breeding pairs by breeding mice carrying the $G b a^{L 444 P}$ allele with mice harboring the knockout glucosylceramide synthase $(U g c g)$ allele (23), which controls the glucosylceramide synthesis pathway (Figure 1, a and b). Both lines were on mixed C57BL/6, 129/Sv backgrounds. After interbreeding the double heterozygous mice $\left(\mathrm{Gba}^{+/ L 444 P} \mathrm{Ugc}^{+/ K O}\right)$, we were successful in producing adult mice homozygous for the Gba ${ }^{L 444 P}$ allele that were heterozygous for the disrupted $U g c g$ gene $\left(U g c g^{+/ K O}\right)$ (Figure $1 \mathrm{~b}$ ). Through the crossing of these double mutant mice, we found that some $\mathrm{Gba} a^{L 444 P / L 444 P} \mathrm{Ugcg}^{+/+}$pups survived past weaning. Interbreeding these $\mathrm{G} b a^{\mathrm{L} 444 P / \mathrm{L} 444 P} \mathrm{Ugcg}^{+/+}$mice under optimal cage conditions allowed survival of slightly more than half of homozygous mutants past weaning (90 pups out of 167 born).

We have not obtained weaned mutant mice from heterozygous $\left(\mathrm{Gba}^{+/ L 444 P}\right)$ mating pairs (out of 110 mice weaned: $\left.39, \mathrm{Gba}^{+/ L 444 P} ; 71, \mathrm{Gba}^{+/+} ; 0, \mathrm{G} b a^{\mathrm{L} 444 P / L 444 P}\right)$. We infer that the enhanced survival of mutant mice when the mating pairs are homozygous for the mutant allele may be due in part to the absence of competition with their healthy wild-type and heterozygous littermates. In all the experiments described here, the mutant mice were of the genotype $\mathrm{Gba}^{\mathrm{L444P} / \mathrm{L} 444 \mathrm{P}} \mathrm{Ugcg}^{+/+}$.

To ensure similar genetic backgrounds, control wildtype mice were derived from heterozygous matings or from generation and background-matched mating pairs. The mice were maintained under specific pathogen-free conditions.

Blood count and blood chemistry. Blood specimens were taken from the vena cava under deep anesthesia. Whole blood was obtained from six mutant and three wildtype mice at 3 months of age. Serum was obtained from four mutant and four wild-type mice at 2 months of age. Whole blood cell counts and blood chemistry were determined in the Department of Laboratory Medicine at the National Institutes of Health. The activity of angiotensin-converting enzyme (ACE) was determined using a ACE Reagent and Calibrator kits (Sigma Diag-
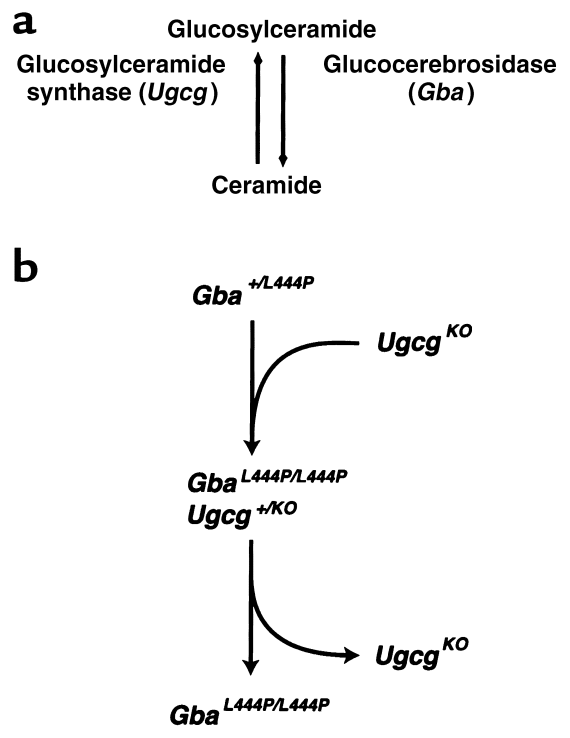

\section{Figure 1}

Degradation and synthesis of glucosylceramide, and derivation of Gba $a^{L 44 P / L 444 P}$ mice. (a) Pathway of glucosylceramide degradation and synthesis. Glucosylceramide synthase is encoded by the Ugcg gene. Glucocerebrosidase (known also as acid $\beta$-glucosidase) is encoded by the Gba gene. (b) Breeding scheme to derive adult Gba $a^{L 444 / L 444}$ mice. As described in Methods, heterozygous mice with the L444P mutation in the Gba gene were cross-bred with mice carrying a disrupted $U g c g$ allele $\left(U g c g^{K O}\right)$ to eventually obtain adult mice with the

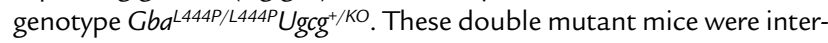
crossed to obtain adult mice with the genotype $\mathrm{Gba}^{L 444 \mathrm{P} / \mathrm{L444P}} \mathrm{Ugcg}^{+/+}$.

nostics, St. Louis, Missouri, USA). Serum chitotriosidase activity was determined as described (24).

Lipid and enzyme analysis. Acid $\beta$-glucosidase activity was determined in tissue extracts as previously described (21). Neutral sphingolipid analysis was accomplished by published methods (25) except that the extraction was for 12 hours at $40^{\circ} \mathrm{C}$. After desalination, the samples were separated into acidic and neutral glycosphingolipids by anion exchange chromatography with DEAE-cellulose (26) with some modifications. The lipid mixture was dispersed in $1 \mathrm{ml}$ of chloroform/methanol/water $(3: 7: 1, \mathrm{vol} / \mathrm{vol} / \mathrm{vol})$ and applied to the columns. The neutral lipids were eluted with $8 \mathrm{ml}$ of the same solvent, and then the acidic lipids were eluted with chloroform/methanol/0.8 M ammonium acetate in water $(3: 7: 1, \mathrm{vol} / \mathrm{vol} / \mathrm{vol})$. The plate was developed according to Shayman et al. (27), and the bands were detected with a phosphoric acid/copper sulfate reagent (25).

Quantitative $m R N A$ analysis by real-time PCR. Cytokine gene expression levels were determined after reverse transcription of RNA samples by real-time PCR by using an ABI PRISM 7700 Sequence Detection System (Applied Biosystems, Foster City, California, USA) as described previously (24). The TaqMan Pre-developed Assay Reagent kits for murine TNF- $\alpha$ and IL-1 $\beta$ (Applied Biosystems) were used. Total RNA was isolated from liver and lymph nodes with TRIzol (GIBCO 
BRL; Life Technologies Inc., Rockville, Maryland, USA). cDNA was synthesized from total RNA with reverse transcriptase reaction using the Superscript II kit (Invitrogen Corp., Carlsbad, California, USA). cDNA was synthesized from $1 \mu \mathrm{g}$ of total RNA for TNF- $\alpha$ and $300 \mathrm{ng}$ of total RNA for IL-1 $\beta$. For standardization of quantitation, GAPDH was amplified simultaneously. The expression level of each gene is presented as fold increase in mutant mice compared with control mice. Experiments were performed with four to seven mice for each genotype.

Histology. After exsanguination of the mice, liver, spleen, brain, lung, skin from the back of the neck, and lymph nodes of cervical, axillary, inguinal, and mesenterial regions were dissected out. The specimens were fixed in $10 \%$ buffered formalin solution, then embedded in paraffin for light microscopic and immunohistochemical studies. Sections ( $5 \mu \mathrm{m}$ thick) were stained with hematoxylin and eosin (H\&E) and periodic acidSchiff (PAS) with diastase digestion. Hepatic cell death was examined on the paraffin sections by in situ TUNEL method using the ApopTag kit (Intergen Co., Purchase, New York, USA). For electron microscopy, small pieces of the liver and lymph nodes were fixed in $2.5 \%$ glutaraldehyde in $0.05 \mathrm{~mol} / 1$ cacodylate buffer (pH 7.4) overnight at $4^{\circ} \mathrm{C}$. Specimens were processed by JEF Enterprises (Brookville, Maryland, USA). For immunostaining, paraffin sections were deparaffinized and rehydrated. Sections were subsequently treated with trypsin for antigen retrieval. Sections were then incubated with primary antibodies overnight at $4{ }^{\circ} \mathrm{C}$, with rat anti-F4/80 antibody (Serotec Ltd., Oxford, United Kingdom) for identification of macrophages, and with rabbit anti-human Ig $\lambda$ light chain (NeoMarkers Inc., Fremont, California, USA) and rat antimouse Ig $\kappa$ light chain (BD Pharmingen, San Diego, California, USA) for identification of Ig-producing cells. The washed sections were incubated with labeled polymer peroxidase-conjugated mouse and rabbit secondary antibodies (DAKO Corp., Carpinteria, California, USA). The peroxidase reaction was visualized by diaminobenzidine and hydrogen peroxidase. IgG levels in plasma were estimated using a mouse IgG ELISA Quantitation kit (Bethyl Laboratories, Inc. Montgomery, Texas, USA).

\section{Results}

As described in Methods, we were first able to produce adult homozygous $G b a^{L 444 P}$ mice through cross-breeding with mice carrying a knockout glucosylceramide synthase (Ugcg) gene (23), which controls the glucosylceramide synthesis pathway (Figure 1, a and b). Through interbreeding these double mutant mice, we were ultimately successful in producing adult mice that were homozygous for the GbaL444P allele and wild-type at the Ugcg locus (Figure 1b). a

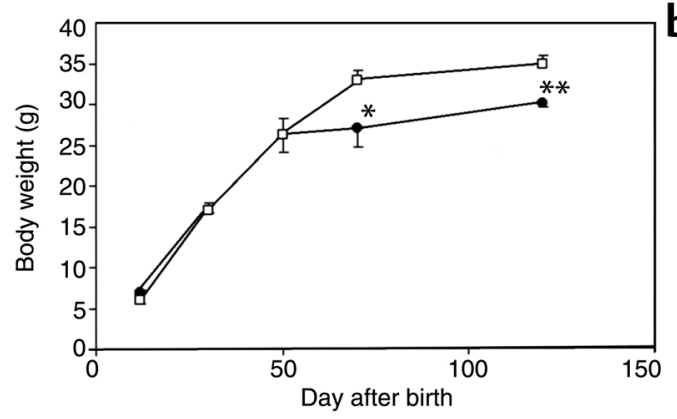

c

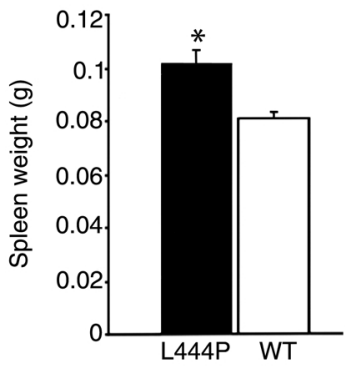

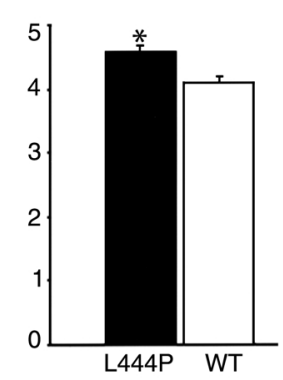

b
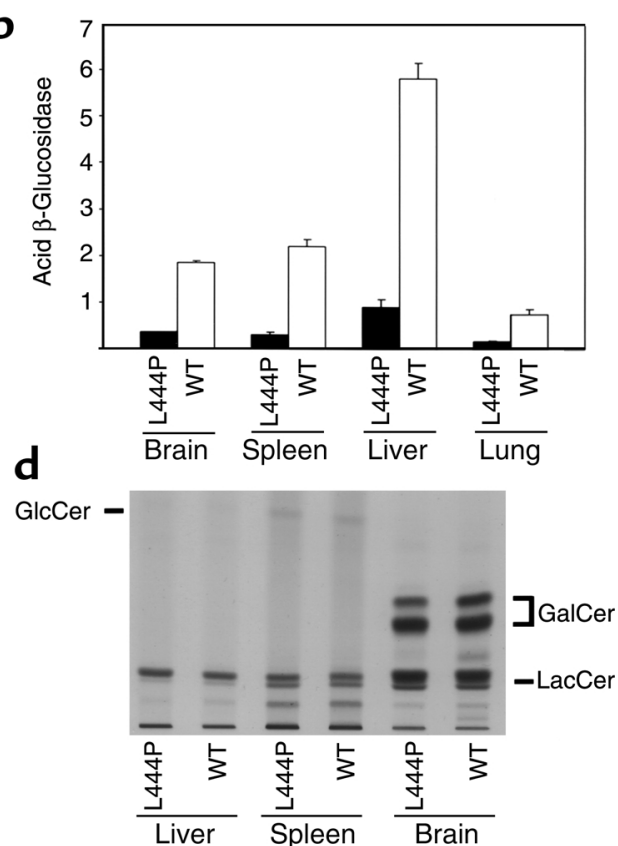

\section{Figure 2}

Growth, enzyme levels, organ weights, and lipid analysis of Gba $a^{L 44 P / L 444 P}$ mice. (a) Body weights were determined from 12 to 120 days of age. Filled circles represent the weights of $G b a^{L 444 P / L 444 P}$ mice; open squares represent the weights of wild-type mice. Results are means $\pm S E M$ for three to seven male mice at each age. ${ }^{*} P<0.05,{ }^{*} P<0.01$. (b) Acid $\beta$-glucosidase activity in brain, spleen, liver, and lung of mutant (L444P) and wild-type (WT) mice. Activity is expressed as nanomoles of substrate cleaved per milligrams of protein per hour. Results are means \pm SEM for three mice each. (c) Left panel: Spleen weights of mutant (L444P) and wild-type (WT) mice. Results are means \pm SEM for 23-24 mice. ${ }^{*} P<0.001$. Right panel: Ratio of liver to body weight of mutant (L444P) and wild-type (WT) mice. ${ }^{*} P<0.01$. Results are means \pm SEM for 21-22 mice. (d) Thin-layer chromatography of neutral lipid fraction from liver, spleen, and brain of 2-month-old mutant (L444P) and wildtype (WT) mice. The migration positions of glucosylceramide (GlcCer), galactosylceramide (GalCer), and lactosylceramide (LacCer) are shown. 
Table 1

Hematologic profile of Gba ${ }^{L 44 P / L 444 P}$ mice

\begin{tabular}{lcc}
\hline & Wild-type & Gba ${ }^{L 444 P / L 444 P}$ \\
WBC $(\mathrm{K} / \mu \mathrm{L})$ & 10.5 & $6.5^{\mathrm{A}}$ \\
$\mathrm{RBC}(\mathrm{M} / \mathrm{ml})$ & 11.6 & $10.3^{\mathrm{B}}$ \\
$\mathrm{Hb}(\mathrm{g} / \mathrm{dl})$ & 17.0 & $15.6^{\mathrm{A}}$ \\
$\mathrm{Ht}(\%)$ & 55.6 & $49.4^{\mathrm{A}}$ \\
$\mathrm{Plt}(\mathrm{K} / \mu \mathrm{l})$ & 1,167 & 1,247 \\
$\mathrm{Chol}(\mathrm{mg} / \mathrm{dl})$ & 157 & $80^{\mathrm{B}}$ \\
$\mathrm{AST}(\mathrm{U} / \mathrm{l})$ & 44 & $71^{\mathrm{A}}$ \\
$\mathrm{ALT}(\mathrm{U} / \mathrm{l})$ & 30 & $42^{\mathrm{A}}$ \\
$\mathrm{ACE}(\mathrm{U} / \mathrm{dl})$ & 24 & 29 \\
Chitotriosidase & 167 & $609^{\mathrm{A}}$ \\
Acid phosphatase & 15 & 27
\end{tabular}

Blood and serum samples were analyzed for white blood cell count (WBC), red blood cell count $(\mathrm{RBC})$, hemoglobin $(\mathrm{Hb})$, hematocrit $(\mathrm{Ht})$, platelet count (Plt), total cholesterol (Chol.), aspartate aminotransferase (AST), alanine aminotransferase (ALT), and ACE. The activity of chitotriosidase and acid phosphatase is expressed as nmol substrate cleaved per $\mathrm{mg}$ protein per hour. $n=3-6 .{ }^{A} P<0.05,{ }^{B} P<0.0001 . K, 10^{3} ; \mathrm{M}, 10^{6}$.

Survival, growth, viability, and organ size. Only about half of the Gba $a^{L 444 / L 444 P}$ mice survived past weaning. These survivors were generally fertile and had a lifespan of more than a year. The body weight of the mutant mice was normal until 50 days of age. After that time, the body weight plateaued at about $15 \%$ less than that of age-matched controls (Figure 2a). The liver and spleen weights of the mutant mice were slightly enlarged when compared with those of normal mice (Figure 2c).

Acid $\beta$-glucosidase activity and glucosylceramide levels. The activity of acid $\beta$-glucosidase in several organs of the mutant mice was found to be $15-20 \%$ of normal activity (Figure 2b). Comparison of enzyme levels with those of mice homozygous for the more severe $G b a^{R e c N c i}$ mutation indicates that at least half of the $\beta$-glucosidase activity in the $G b a^{L 444 P}$ mutant mice is the result of residual glucocerebrosidase activity (21). Abnormal glucosylceramide accumulation in the liver, spleen, or brain of mutant mice was not detectable by thin-layer chromatography analysis (Figure 2d). Mice analyzed ranged in age from 2 months to over 1 year old.

Hematologic findings and blood chemistry. The red blood cell counts of the mutant mice were $88.8 \%$ of control values with hemoglobin and hematocrit values at $91.8 \%$ and $88.7 \%$, respectively (Table 1 ). White blood cell counts in mutant mice were $62 \%$ of control. Platelet counts were not significantly depressed in the mutant mice.

Total serum cholesterol in mutant mice was significantly lower than in controls (Table 1). The liver enzymes aspartate aminotransferase and alanine aminotransferase were $160 \%$ and $141 \%$ of control levels, respectively, indicating liver damage (Table 1$)$. Chitotriosidase, an enzyme that is massively elevated in the serum of Gaucher disease patients (28), was modestly elevated in the serum of the mutant mice. However, acid phosphatase and ACE, two other serum enzymes commonly elevated in the patients, were not significantly altered in the mutant mice.
Histology. The major histologic finding in the Gba $a^{L 44 P / L 444 P}$ mice was multi-organ system inflammation. In mutant mice, inflammatory foci were found in the liver (Figure 3, a and b), while no inflammatory lesions were noted in the livers of control mice. The inflammatory foci in the livers of mutant mice were detected as early as 1 month of age. These lesions were scattered throughout the liver and contained macrophages, lymphocytes, and neutrophils. Apoptotic hepatocytes could be identified in the inflammatory lesions by the TUNEL method (Figure 3e). Kupffer cells in control mice had a ramified structure (Figure 3c), while in mutant mice the Kupffer cells were amoeboid in shape, a morphology associated with an activated state (Figure 3d) (24). mRNA for the proinflammatory cytokine TNF- $\alpha$ was elevated about threefold in the liver of the mutant mice relative to controls (Figure 3f). Swollen, multinucleated macrophages containing brown cytoplasmic granules were identified in the liver of a 7 -month-old mutant mouse (Figure 4, inset). These cells were about $20 \mu \mathrm{m}$ in diameter and found both in inflammatory foci and in sinusoids. Electron microscopy showed lipofuscin granules and abundant
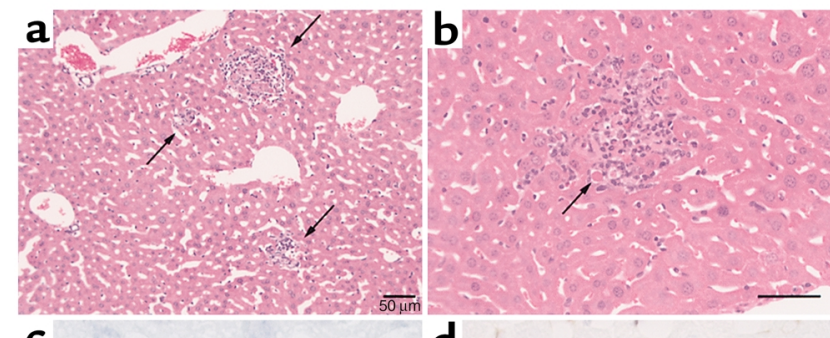

C
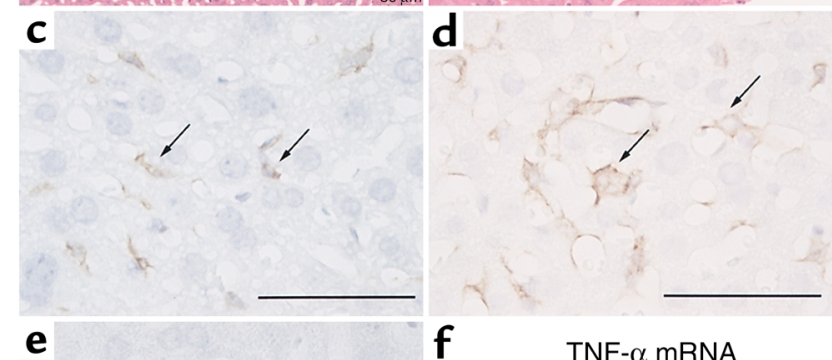

f

TNF- $\alpha$ mRNA
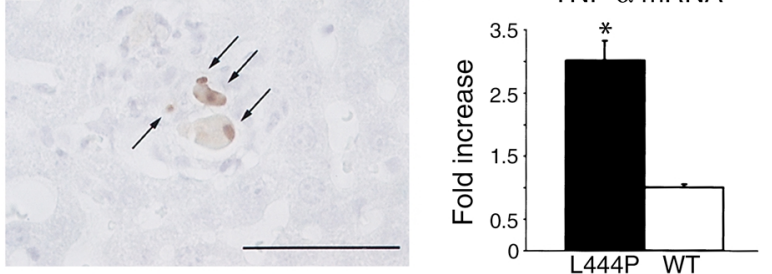

Figure 3

Inflammatory reaction in the liver of Gba ${ }^{L 444 P / L 444 P}$ mice. (a) Inflammatory foci were scattered in the liver of a 2-month-old mutant mouse (arrows). (b) Higher magnification of an inflammatory area. Note the apoptotic hepatocyte in the inflammatory area (arrow). (c) Immunostaining with $\mathrm{F} 4 / 80$ antibody to visualize ramified Kupffer cells in wild-type liver sections. (d) In mutant liver, the F4/80-positive Kupffer cells were amoeboid and swollen. (e) TUNEL-positive, apoptotic nuclei (arrows) were distinguished in the inflammatory lesion of the mutant mouse. (f) TNF- $\alpha$ mRNA expression in the liver of mutant and wild-type mice. ${ }^{*} P<0.001$. Results are means \pm SEM for four to seven mice each. Bars $=50 \mu \mathrm{m}$. 


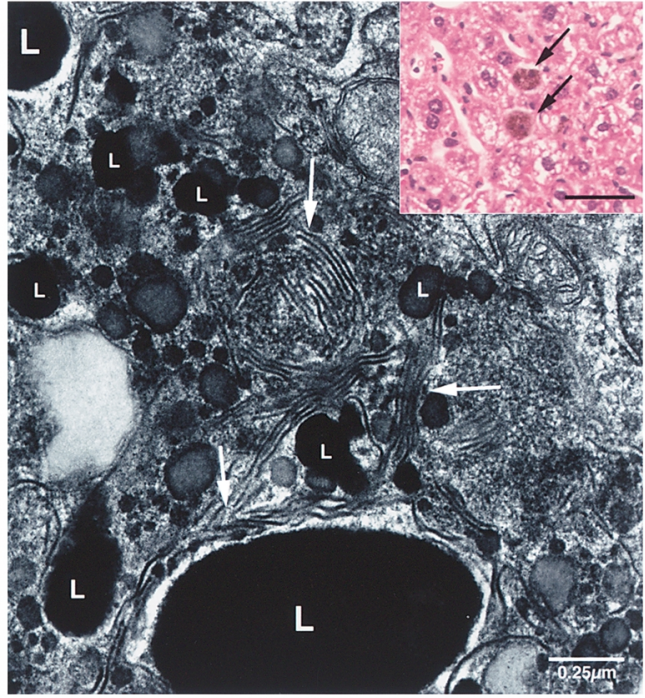

\section{Figure 4}

Abnormal macrophages with lipofuscin granules and tubular structures. Inset; liver section from a 7-month-old mutant mouse showing multinucleated macrophages (arrows) containing abundant brown granules in their cytoplasm. Bar $=50 \mu \mathrm{m}$. Main portion: Electron microscopy revealed that these cells contain lipofuscin granules (L) and abundant parallel and twisted tubular structures (arrows) in their cytoplasm. Bar $=0.25 \mu \mathrm{m}$.

tubular structures in the cytoplasm (Figure 4). The tubules were in parallel or twisted arrays, as has been described for the structure of stored intracellular glucosylceramide (29).

Inflammation was also noted in the spleen and lung. Aggregations of inflammatory cells, consisting of neutrophils, lymphocytes, and macrophages, were found in the lungs of mutant mice (Figure 5a). Excessive neutrophil infiltration also appeared in the red pulp of the spleen of the mutant mice (not shown).

Histologic abnormalities were noted in skin of the Gba $a^{L 444 P / L 444 P}$ mice, as has been previous described (21). Both the stratum corneum and the epidermis were thickened in the mutant mice (Figure 5c). Infiltration of inflammatory cells was also noted in the upper dermis in mutant mice (Figure 5c).

Mutant mice exhibited lymphadenitis; cervical, axillary, inguinal, and mesenteric lymph nodes were affected (Figure 6a). Follicular hyperplasia and distended parafollicular areas were observed in the mutant mice (Figure 6b). Abnormally large numbers of plasma cells were also found in the lymph nodes of mutant mice, consistent with plasmacytosis (Figure $6 \mathrm{f}$ ). These cells were PAS-positive (not shown) and reacted with antibodies to $K$ and $\lambda$ light chains (Figure $6 \mathrm{~d})$. By electron microscopy, these cells had a characteristic plasma cell morphology with abundant, dilated endoplasmic reticulum containing granular material. In the lymph nodes of mutant mouse IL-1 $\beta$, mRNA was elevated fourfold above control levels (Figure $6 \mathrm{~g})$. Serum IgG in mutant mice was significantly higher than in control mice (Figure 6g).

No Gaucher-like cells of characteristic "wrinkled tissue paper" appearance (1) were found in liver, spleen, bone marrow, lymph nodes, lung, brain, or any other tissues of the mutant mice. No histologic abnormalities of the bones or CNS were observed. The mice examined ranged in age from 2 months to over 1 year.

\section{Discussion}

In Gaucher disease patients, macrophages become engorged with glucosylceramide and are found in nearly every organ. These Gaucher cells are believed to contain the bulk of the glucosylceramide storage in tissues and to be the proximate cause of the characteristic hepatosplenomegaly and possibly the bony deterioration. However, many other clinical manifestations have been described for which the etiology is unclear due in part to the absence of viable animal models.

Mice homozygous for the $G b a^{L 444 P}$ mutation had levels of acid $\beta$-glucosidase activity in the range of type 1 patients but did not accumulate large amounts of glucosylceramide in tissues or show the presence of clas-

\section{Figure 5}

Pathology in lung and skin of Gba ${ }^{L 444 / L 444 P}$ mice. (a) Lung of mutant mice showed scattered inflammatory cell aggregations (arrows) in the mesenchyme. (b) Lung of wild-type mice showed no signs of inflammation. (c and d) Skin sections of mutant (c) and control mice (d). Stratum corneum (SC) and epidermis (ED) of mutant mice were thicker than those of wild-type mice. Inflammatory cell infiltration was distinguished in upper dermis (DER) of mutant mice (arrows). Bars $=50 \mu \mathrm{m}$.

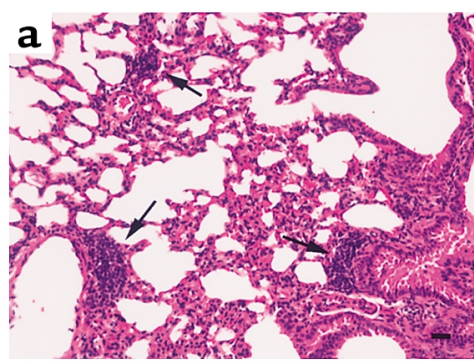

C

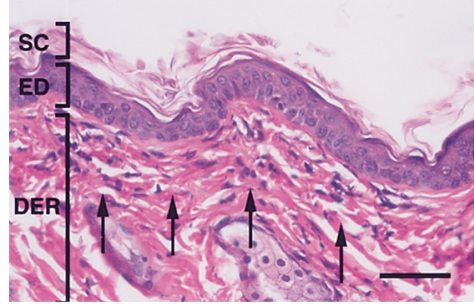




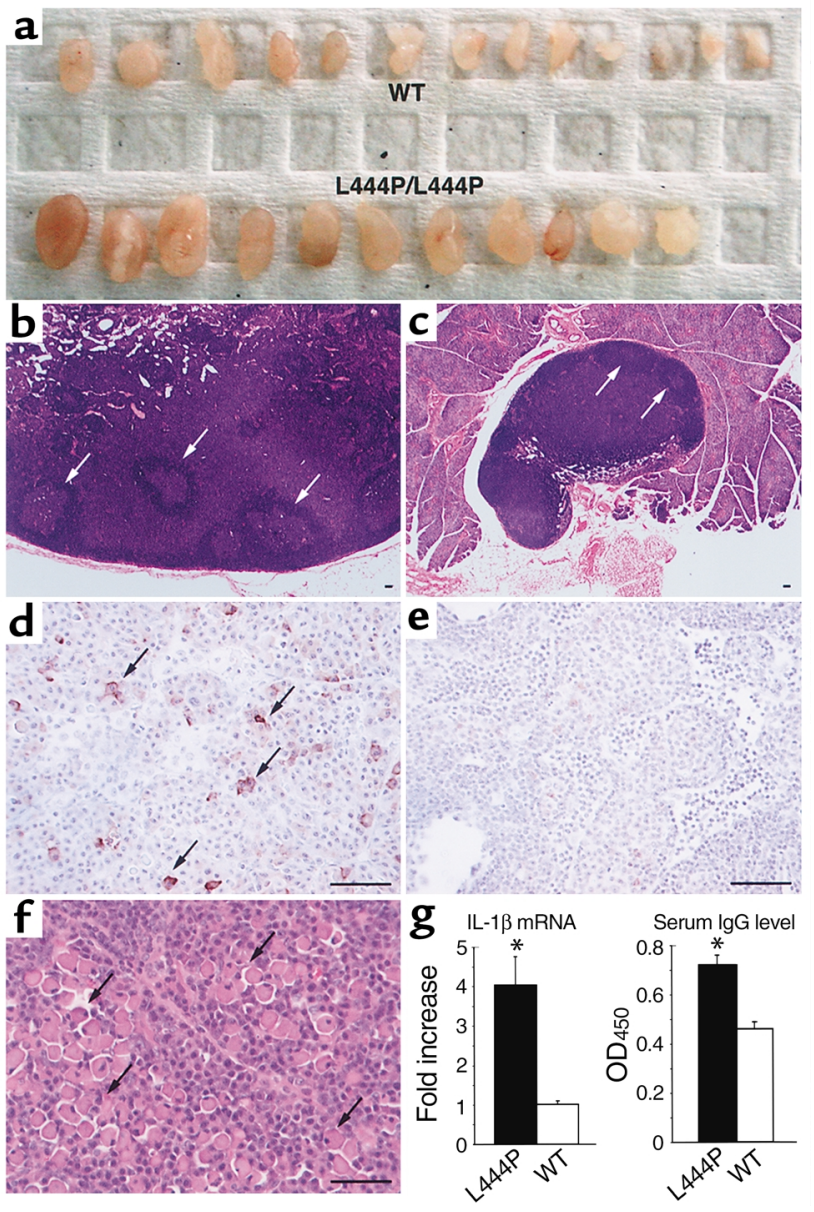

Figure 6

Lymph node hypertrophy in the Gba ${ }^{L 444 P / L 444 P}$ mice. (a) Cervical, axillary, inguinal, and mesenteric lymph nodes were removed from a 2month-old wild-type and a 2-month-old Gba ${ }^{L 444 P / L 444 P}$ mouse. The lymph nodes of mutant mice (b) showed follicular hyperplasia and dilated parafollicular zones compared with the lymph nodes of control mice (c). Immunostaining for $\kappa$ light chain showed large numbers of positive cells in the lymph nodes of the mutant mice (d) compared with wild-type mice (e). Arrows point out a few of the positive cells. $(\mathbf{f})$ Plasmacytosis in the cervical lymph node of a 9-month-old mutant mouse. Arrows point out a few of the abundant large plasma cells. Bars $=50 \mu \mathrm{m}$. (g) Left panel: IL-1 $\beta$ mRNA expression in the cervical lymph nodes of mutant ( $L 444 \mathrm{P})$ and wild-type mice. Results are means \pm SEM for six mice. ${ }^{*} P<0.05$. Right panel: Serum IgG levels in mutant (L444P) and wild-type mice. Results are means \pm SEM for five mice.

sic Gaucher cells. Yet even without these hallmarks of the disease, the mutant mice displayed some features reported in Gaucher disease patients (Table 2). These included systemic inflammation, anemia and leukopenia, skin abnormalities, and depressed serum cholesterol levels.

Although Gaucher-like cells of classic morphology were not identified in the mutant mice, there were detectable alterations in the macrophage population. Changes in macrophage morphology indicative of an activated state were noted in livers. Lowered cholesterol and elevated chitotriosidase activity, which have been attributed to an activated macrophage population in
Gaucher patients $(28,30,31)$, were observed. In the liver, rare macrophages of abnormal morphology were identified with lipofuscin granules, consistent with an impairment in lysosomal degradation. These cells contained the characteristic tubular structures of stored glucosylceramide (29), suggesting that the mice may have limited local glycolipid accumulation. The absence of a large population of storage macrophages likely underlies the lack of detectable glucosylceramide storage in tissues of the mutant mice and may explain the absence of some of the more severe symptomology such as hepatosplenomegaly and bone lesions.

The dominant pathologic feature in the mutant mice was a multisystem inflammatory reaction with inflammatory cell infiltration in several organs, lymphadenopathy, and elevated TNF- $\alpha$ and IL- $1 \beta$ mRNA expression. Evidence of B cell hyperproliferation was found along with elevated serum IgG levels. In patients, Gaucher cells, which have properties of activated macrophages $(9,32)$, typically infiltrate many organs. Cytokines including TNF- $\alpha$ and IL- $1 \beta$ have been reported to be increased in patients (12-15). B cell dysfunction characterized by hypergammaglobulinemia $(10,17)$ and plasmacytosis $(11,33)$ has been described in some patients. An apparently increased risk of B cell malignancies - multiple myeloma and chronic lymphocytic leukemia - has been described as associated with the disease $(9,17-19)$. It has been suggested that the association of these lymphoid cancers with Gaucher disease may result from the chronic stimulation of $B$ cells through a sustained inflammatory reaction $(9,17,18)$.

How might the inflammatory symptoms in the mice be triggered in the absence of large amounts of glucosylceramide storage and Gaucher cells? Recent studies have demonstrated the activity of various sphingolipid molecules as potent stimulators of signaling pathways involved in inflammation $(34,35)$. G protein-coupled receptors for sphingolipids have been shown to regulate the activity of cells of the immune system $(35,36)$. We suggest that low levels of glucosylceramide or relat-

\section{Table 2}

Comparison of some clinical features of type 1 Gaucher disease and the Gba $a^{L 444 P / L 444 P}$ mouse

\section{Gaucher disease, type 1}

$G b a^{L 444 P / L 444 P}$

$\beta$-Glucosidase deficiency

Tissue GlcCer accumulation

Hepatosplenomegaly

Gaucher cells

Bone lesions

Lymphadenopathy

Elevated serum IgG

Elevated plasma cells

Elevated TNF- $\alpha$, IL-1 $\beta$

Elevated chitotriosidase

Low serum cholesterol

Skin abnormalities

Anemia

Leukocytopenia

Thrombocytopenia

GlcCer, glucosylceramide. 
ed glycosphingolipids, generated focally in the mice, may act as signaling molecules to dysregulate the immune system. It is of interest that TDAG8, a G protein-coupled receptor found on macrophages, is triggered by glucosylsphingosine (36).

The presence of inflammatory pathology in the $G b a^{L 444 P}$ mutant mice suggests that inflammation can be triggered with minimal glucosylceramide storage. This inflammation reaction included a hyperproliferative response of B cells, a feature reported in Gaucher patients. The results raise the possibility that even "asymptomatic" patients - those without large amounts of lipid storage - may face some risk from a sustained inflammatory response.

\section{Acknowledgments}

We thank Soroku Yagihashi (Department of Pathology, Hirosaki University School of Medicine) for help with interpretation of the pathology in the mutant mouse. This work was supported in part by Deutsche Forschungsgemeinschaft Grant SFB 400.

1. Beutler, E., and Grabowski, G.A. 2001. Gaucher disease. In The metabolic and molecular bases of inherited disease. Volume 3. C.R. Scriver, A.L. Beaudet, W.S. Sly, and D. Valle, editors. McGraw-Hill. New York, New York, USA. 3635-3668.

2. Brady, R.O., Pentchev, P.G., Gal, A.E., Hibbert, S.R., and Dekaban, A.S. 1974. Replacement therapy for inherited enzyme deficiency. Use of purified glucocerebrosidase in Gaucher's disease. N. Engl. J. Med. 291:989-993.

3. Beutler, E., Dale, G.L., Guinto, D.E., and Kuhl, W. 1977. Enzyme replacement therapy in Gaucher's disease: preliminary clinical trial of a new enzyme preparation. Proc. Natl. Acad. Sci. USA. 74:4620-4623.

4. Barton, N.W., et al. 1991. Replacement therapy for inherited enzyme deficiency: macrophage-targeted glucocerebrosidase for Gaucher's disease. N. Engl. J. Med. 324:1464-1470.

5. Cox, T., et al. 2000. Novel oral treatment of Gaucher's disease with N-butyldeoxynojirimycin (OGT 918) to decrease substrate biosynthesis. Lancet. 355:1481-1485.

6. Abe, A., Wild, S.R., Lee, W.L., and Shayman, J.A. 2001. Agents for the treatment of glycosphingolipid storage disorders. Curr. Drug Metab. 2:331-338.

7. Tifft, C.J., and Proia, R.L. 2000. Stemming the tide: glycosphingolipid synthesis inhibitors as therapy for storage diseases. Glycobiology. 10:1249-1258. 8. Platt, F.M., et al. 2001. Inhibition of substrate synthesis as a strategy for glycolipid lysosomal storage disease therapy. J. Inherit. Metab. Dis. 24:275-290.

9. Cox, T.M. 2001. Gaucher disease: understanding the molecular pathogenesis of sphingolipidoses. J. Inherit. Metab. Dis. 24:106-121.

10. Marti, G.E., et al. 1988. Polyclonal B-cell lymphocytosis and hypergammaglobulinemia in patients with Gaucher disease. Am. J. Hematol. 29:189-194.

11. Marie, J.P., Tulliez, M., Tricottet-Paczinski, V., Reynes, M., and Diebold, J. 1982. Gaucher's disease with monoclonal gammopathy. Significance of splenic plasmacytosis. Scand. J. Haematol. 28:54-58.

12. Barak, V., et al. 1999. Cytokines in Gaucher's disease. Eur. Cytokine Netw. 10:205-210.

13. Lichtenstein, M., Zimran, A., and Horowitz, M. 1997. Cytokine mRNA in Gaucher disease. Blood Cells Mol. Dis. 23:395-401.
14. Allen, M.J., Myer, B.J., Khokher, A.M., Rushton, N., and Cox, T.M. 1997. Pro-inflammatory cytokines and the pathogenesis of Gaucher's disease: increased release of interleukin-6 and interleukin-10. QJM. 90:19-25.

15. Michelakakis, H., et al. 1996. Plasma tumor necrosis factor- $\alpha$ (TNF- $\alpha)$ levels in Gaucher disease. Biochim. Biophys. Acta. 1317:219-222.

16. Garfinkel, D., et al. 1985. Multiple plasmocytomas of the liver and the spleen. Clin. Nucl. Med. 10:819.

17. Shoenfeld, Y., et al. 1982. Gaucher's disease: a disease with chronic stimulation of the immune system. Arch. Pathol. Lab. Med. 106:388-391.

18. Burstein, Y., Rechavi, G., Rausen, A.R., Frisch, B., and Spirer, Z. 1985. Association of Gaucher's disease and lymphoid malignancy in 2 children. Scand. J. Haematol. 35:445-447.

19. Fox, H., McCarthy, P., Andre-Schwartz, J., Shoenfeld, Y., and Miller, K.B. 1984. Gaucher's disease and chronic lymphocytic leukemia. Possible pathogenetic link between Gaucher's disease and B-cell proliferations? Cancer. 54:312-314.

20. Tybulewicz, V.L.J., et al. 1992. Animal model of Gaucher's disease from targeted disruption of the mouse glucocerebrosidase gene. Nature. 357:407-410.

21. Liu, Y., et al. 1998. Mice with type 2 and 3 Gaucher disease point mutations generated by a single insertion mutagenesis procedure. Proc. Natl. Acad. Sci. USA. 95:2503-2508.

22. Holleran, W.M., et al. 1994. Consequences of beta-glucocerebrosidase deficiency in epidermis. Ultrastructure and permeability barrier alterations in Gaucher disease. J. Clin. Invest. 93:1756-1764.

23. Yamashita, T., et al. 1999. A vital role for glycosphingolipid synthesis during development and differentiation. Proc. Natl. Acad. Sci. USA. 96:9142-9147.

24. Wada, R., Tifft, C.J., and Proia, R.L. 2000. Microglial activation precedes acute neurodegeneration in Sandhoff disease and is suppressed by bone marrow transplantation. Proc. Natl. Acad. Sci. USA. 97:10954-10959.

25. Sango, K., et al. 1996. Mice lacking both subunits of lysosomal beta-hexosaminidase display gangliosidosis and mucopolysaccharidosis. Nat. Genet. 14:348-352.

26. Momoi, T., Ando, S., and Magai, Y. 1976. High resolution preparative column chromatographic system for gangliosides using DEAESephadex and a new porus silica, Iatrobeads. Biochim. Biophys. Acta. 441:488-497.

27. Shayman, J.A., et al. 1991. Modulation of renal epithelial cell growth by glucosylceramide. Association with protein kinase C, sphingosine, and diacylglycerol. J. Biol. Chem. 266:22968-22974.

28. Hollak, C.E., van Weely, S., van Oers, M.H., and Aerts, J.M. 1994. Marked elevation of plasma chitotriosidase activity. A novel hallmark of Gaucher disease. J. Clin. Invest. 93:1288-1292.

29. Lee, R.E. 1968. The fine structure of the cerebroside occurring in Gaucher's disease. Proc. Natl. Acad. Sci. USA. 61:484-489.

30. Ginsberg, H., et al. 1984. Reduced plasma concentrations of total, low density lipoprotein and high density lipoprotein cholesterol in patients with Gaucher type I disease. Clin. Genet. 26:109-116.

31. Le, N.A., Gibson, J.C., Rubinstein, A., Grabowski, G.A., and Ginsberg, H.N. 1988. Abnormalities in lipoprotein metabolism in Gaucher type 1 disease. Metabolism. 37:240-245.

32. Moran, M.T., et al. 2000. Pathologic gene expression in Gaucher disease: up-regulation of cysteine proteinases including osteoclastic cathepsin K. Blood. 96:1969-1978.

33. Kaloterakis, A., et al. 1999. Systemic AL amyloidosis in Gaucher disease. A case report and review of the literature. J. Intern. Med. 246:587-590.

34. Kabarowski, J.H., Zhu, K., Le, L.Q., Witte, O.N., and Xu, Y. 2001. Lysophosphatidylcholine as a ligand for the immunoregulatory receptor G2A. Science. 293:702-705.

35. Le, L.Q., et al. 2001. Mice lacking the orphan G protein-coupled receptor G2A develop a late-onset autoimmune syndrome. Immunity. 14:561-571.

36. Im, D.S., Heise, C.E., Nguyen, T., O’Dowd, B.F., and Lynch, K.R. 2001. Identification of a molecular target of psychosine and its role in globoid cell formation. J. Cell Biol. 153:429-434. 